04.2

\title{
Расчет нейтронного выхода токамака Глобус-М2 с учетом анизотропии по скоростям функции распределения ионов при нейтральной инжекции атомов высокой энергии
}

\author{
(C) О.М. Скрекель, Н.Н. Бахарев, В.К. Гусев, Е.О. Киселев \\ Физико-технический институт им. А.Ф. Иоффе РАН, Санкт-Петербург, Россия \\ E-mail: fosa97@gmail.com
}

Поступило в Редакцию 17 сентября 2020 г.

В окончательной редакции 8 ноября 2020 г.

Принято к публикации 8 ноября 2020 г.

Рассматриваются два алгоритма расчета нейтронного выхода токамака Глобус-М2 для случая анизотропных по скоростям функций распределения ионов. Такие расчеты необходимы для оценки потока нейтронов, рожденных в результате взаимодействия между быстрыми частицами (дейтронами), возникающими при инжекции пучка атомов высокой энергии. Первый алгоритм представляет собой вычисление шестимерного интеграла в пространстве скоростей, а второй использует разложение функции распределения ионов по полиномам Лежандра, что позволяет уменьшить размерность интегрирования. Проводится сравнение результатов, полученных при помощи описанных алгоритмов, и обсуждаются достоинства и недостатки каждого из них.

Ключевые слова: нейтронный выход, сферический токамак, Глобус-М2.

DOI: 10.21883/PJTF.2021.04.50640.18548

Компактные сферические токамаки могут быть использованы в качестве драйвера в гибридных схемах, где термоядерный источник нейтронов (ТИН) окружен зоной делящегося материала. Такие гибридные реакторы помогут решить проблему „замыкания ядерного топливного цикла“, поскольку будут не только вырабатывать энергию, но и позволят нарабатывать новое и перерабатывать отработанное ядерное топливо. Кроме того, создание компактных ТИН ускорит развитие классических термоядерных реакторов, так как даст возможность проводить исследования материалов при значительных потоках термоядерных нейтронов. Цель настоящей работы - рассмотрение двух алгоритмов расчета функции источника нейтронов, рожденных в результате взаимодействия высокоэнергетических ионов друг с другом, а также сравнение работы этих алгоритмов при характерных условиях эксперимента на токамаке Глобус-М2.

В 2018 г. была проведена реконструкция компактного сферического токамака Глобус-М [1-3], включающая в себя модернизацию электромагнитной системы: во время первой экспериментальной кампании в 2019 г. тороидальное магнитное поле было увеличено с 0.5 до $0.8 \mathrm{~T}$, а ток плазмы — с 250 до $400 \mathrm{kA} \mathrm{[4].} \mathrm{Это}$ позволило существенно повысить параметры плазмы и приблизить их к планируемым в будущих ТИН. Основной вклад в нейтронный выход на токамаках Глобус-М/М2 приходится на взаимодействие быстрых частиц, возникающих при инжекции пучка атомов высокой энергии, и ионов основной плазмы. Вследствие увеличения тороидального магнитного поля и тока плазмы улучшилось удержание высокоэнергетических ионов, что привело к росту полного нейтронного потока [4].
При этом также существенно возросла доля нейтронов, рожденных в результате взаимодействия быстрых частиц друг с другом. В связи с этим данное взаимодействие необходимо учитывать при сравнении рассчитываемого нейтронного выхода с экспериментальным.

Распределение ионов плазмы может быть представлено в виде суммы двух составляющих.

1. Максвелловское распределение ионов основной плазмы, которое восстанавливается на основе измерений ионной температуры, выполненных с помощью анализатора атомов перезарядки или активной спектроскопической диагностики [5], пространственного распределения концентрации электронов, измеренного при помощи диагностики томсоновского рассеяния [6], данных диагностики эффективного заряда [7].

2. Анизотропное по скоростям распределение быстрых ионов, которое не описывается аналитически. Для его расчета на токамаке Глобус-М2 используются коды [8-10], указанные в таблице.

Скорость реакции ядерного синтеза при взаимодействии частиц двух сортов определяется формулой

$$
\begin{gathered}
Y=\frac{n_{a} n_{b}}{1+\delta_{a b}} R_{a b}, \\
R_{a b}=\int \sigma_{f}(v) v f_{a}\left(\mathbf{v}_{a}\right) f_{b}\left(\mathbf{v}_{b}\right) d \mathbf{v}_{a} d \mathbf{v}_{b},
\end{gathered}
$$

где $R_{a b}$ - удельная скорость реакции, $n_{a}, n_{b}$ - плотности топливных ядер, $v=\left|\mathbf{v}_{a}-\mathbf{v}_{b}\right|-$ модуль относительной скорости частиц, $f_{a}\left(\mathbf{v}_{a}\right), f_{b}\left(\mathbf{v}_{b}\right)-$ функции распределения ионов, нормированные на единицу, $\sigma_{f}(v)-$ сечение реакции синтеза. Дельта-символ Кронекера $\delta_{a b}$ обеспечивает деление на 2 при взаимодействии ядер одного сорта. 
Используемые на токамаке Глобус-М2 коды для расчета анизотропной по скоростям функции распределения быстрых частиц, возникающих при инжекции пучка атомов высокой энергии

\begin{tabular}{c|c}
\hline Код & Рассчитываемые функции распределения ${ }^{* *}$ \\
\hline NUBEAM [8] & $f(r, z, E, \vartheta), f\left(\rho_{t o r}, \varphi, E, \vartheta\right)$ \\
ASCOT [9] & $f(r, z, E, \vartheta), f\left(\rho_{p o l}, \varphi, E, \vartheta\right), f\left(r, z, v_{r}, v_{\theta}, v_{z}\right), f\left(\rho_{p o l}, v_{r}, v_{z}, v_{\theta}\right)$ \\
3D tracking + Boltzmann* $[10]$ & $f\left(\rho_{p o l}, E, \vartheta\right)$
\end{tabular}

* 3D tracking + Boltzmann - трехмерный алгоритм, вычисляющий траектории частиц, объединенный с решением уравнения Больцмана для описания их замедления.

** $r$ - координата частицы в плазме по большому радиусу токамака, $z$ - координата частицы в плазме по высоте от экваториальной плоскости токамака, $E$ - энергия частиц, $\vartheta-$ питч-угол (угол между вектором магнитного поля и вектором скорости частицы), $\varphi$ - полоидальный угол, $\theta$ - тороидальный угол, $v_{r}, v_{z}, v_{\theta}$ - векторы скорости частиц, $\rho_{t o r}=\sqrt{\left(\Phi-\Phi_{0}\right) /\left(\Phi_{b r y}-\Phi_{0}\right)}$ и $\rho_{p o l}=\sqrt{\left(\Psi-\Psi_{0}\right) /\left(\Psi_{b r y}-\Psi_{0}\right)}-$ относительные магнитные координаты, где $\Phi$ - тороидальный магнитный поток, $\Phi_{0}$ - тороидальный магнитный поток на магнитной оси, $\Phi_{b r y}-$ тороидальный магнитный поток на границе плазмы, $\Psi$ - полоидальный магнитный поток, $\Psi_{0}$ - полоидальный магнитный поток на магнитной оси, $\Psi_{b r y}-$ полоидальный магнитный поток на границе плазмы.

Рассмотрим два алгоритма для расчета функции источника нейтронов, возникающих при взаимодействии между быстрыми частицами, с учетом анизотропии их функций распределения по скоростям. Первый алгоритм заключается в непосредственном интегрировании выражения (2). Если функция распределения зависит от модуля скорости и питч-угла (как, например, в случае кода NUBEAM [8] или трехмерного алгоритма, вычисляющего траектории частиц, объединенного с решением уравнения Больцмана для описания их замедления [10]), то она должна быть преобразована к трехмерному распределению по скорости. При данном преобразовании предполагается равномерное распределение вектора скорости частицы вокруг линии магнитного поля при заданном значении питч-угла. Достоинством данного метода расчета является его гибкость: возможность работать с функциями распределения любого вида и данными с высоким уровнем статистических шумов, недостатком - значительное время работы, поскольку для каждой точки сетки вычисляется интеграл в шестимерном пространстве.

Второй алгоритм заключается в преобразовании формулы (2) для удельной скорости реакции к следующему виду [11]:

$$
\begin{aligned}
R_{12}= & 8 \pi^{2} \sum_{n=0}^{\infty} \frac{1}{2 n+1} \int_{0}^{\infty} v_{1} a_{n}\left(v_{1}\right) d v_{1} \int_{0}^{\infty} v_{2} b_{n}\left(v_{2}\right) d v_{2} \\
& \times \int_{\left|v_{1}-v_{2}\right|}^{v_{1}+v_{2}} \sigma_{f}(v) v^{2} P_{n}\left(\frac{v_{1}^{2}+v_{2}^{2}-v^{2}}{2 v_{1} v_{2}}\right) d v
\end{aligned}
$$

В выражении (3) используется разложение функций распределения по полиномам Лежандра

$$
\begin{aligned}
& f_{1}\left(v_{1}, \cos \vartheta_{1}\right)=\sum_{m=0}^{\infty} a_{m}\left(v_{1}\right) P_{m}\left(\cos \vartheta_{1}\right) \\
& f_{2}\left(v_{2}, \cos \vartheta_{2}\right)=\sum_{k=0}^{\infty} b_{k}\left(v_{2}\right) P_{k}\left(\cos \vartheta_{2}\right)
\end{aligned}
$$

где $v_{1,2}$ - модули скоростей ядер сортов 1 и 2, $\cos \vartheta_{1,2}$ - косинусы полярных углов. Таким образом, второй алгоритм расчета функции нейтронного выхода сводится к вычислению суммы со слагаемыми в виде тройных интегралов. Данный способ намного быстрее предыдущего (2), так как в каждой точке плазменного шнура не требует интегрирования произведений трехмерных функций распределения. Однако он применим только для работы с функциями распределения определенного вида, а также требует тщательного подбора параметров сглаживания входных данных из-за использования разложений по полиномам Лежандра.

На рис. 1, a приведен пример распределения плотности быстрых ионов (с энергиями $>5 \mathrm{keV}$ ) в разряде \#38095 на $180 \mathrm{~ms}$, рассчитанный при помощи кода NUBEAM. Основные параметры разряда: тороидальное магнитное поле $B_{T}=0.7 \mathrm{~T}$, ток плазмы $I_{p}=290 \mathrm{kA}$, среднехордовая плотность $\left\langle n_{e}\right\rangle_{l}=8.3 \cdot 10^{19} \mathrm{~m}^{-3}$, плазма изотопов водорода в соотношении $\mathrm{D} / \mathrm{H}=80 / 20 \%$, эффективный заряд $Z_{e f f}=1.6$, основная примесь углерод, инжектировался дейтериевый пучок с энергией $28 \mathrm{keV}$ мощностью $800 \mathrm{~kW}$. Соответствующая функция источника нейтронов $Y(r, z)(1)$, рожденных в процессе взаимодействия высокоэнергетических частиц друг с другом, вычисленная при помощи первого алгоритма (2), представлена на рис. $1, b$. При расчетах предполагалась тороидальная симметрия.

Для сравнения двух алгоритмов расчета рассматривался нейтронный выход $Y(r, z)$, рассчитанный при помощи (2) и (3), в разрядах \#36620, 37067, 37069 , 37070, 37893, 38089, 38095, 38516. Основные параметры данных разрядов изменялись в следующих диапазонах: $B_{T}=0.4-0.7 \mathrm{~T}, I_{p}=180-330 \mathrm{kA}$, $\left\langle n_{e}\right\rangle_{l}=(3.2-8.3) \cdot 10^{19} \mathrm{~m}^{-3}$, инжектировался дейтериевый пучок с энергиями 26-28 keV мощностью 650-800 kW. Сравнение показало высокую степень соответствия результатов двух алгоритмов (рис. 2). Среднее стандартное отклонение результатов расчета равно $\sigma \approx 1.7 \%$.

На данный момент на установке Глобус-М2 нейтронный выход от взаимодействия быстрых частиц друг с 

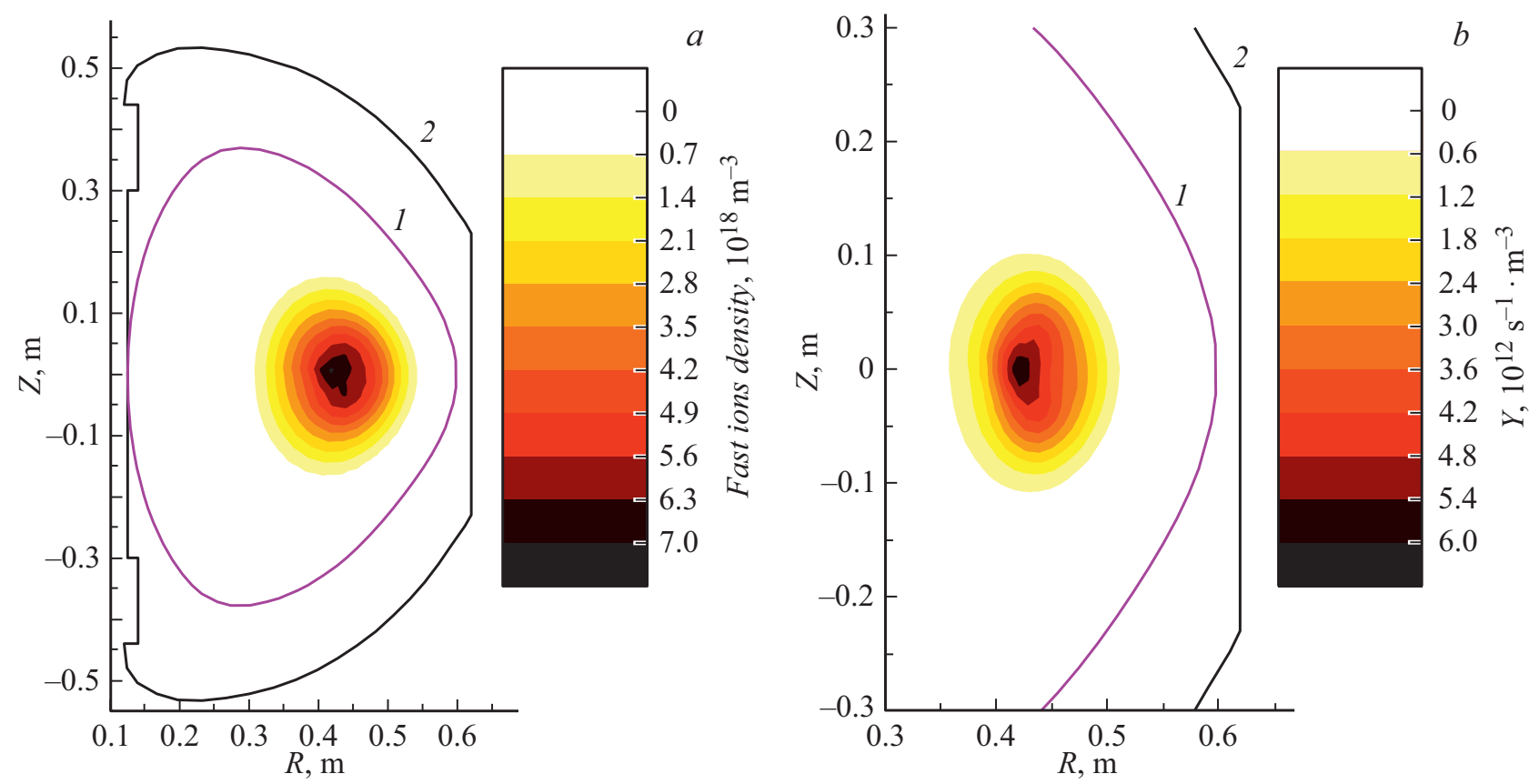

Рис. 1. $a$ - распределение плотности ионов с энергией $>5 \mathrm{keV}$ на токамаке Глобус-М 2 в разряде \#38095 на $180 \mathrm{~ms}$. Рассчитано при помощи кода NUBEAM. $b$ - соответствующая функция источника нейтронов $Y(r, z)(1)$, возникающих в процессе взаимодействия между быстрыми частицами. Рассчитано при помощи алгоритма шестимерного интегрирования в пространстве скоростей (2). 1 -граница плазмы, 2 - граница камеры.

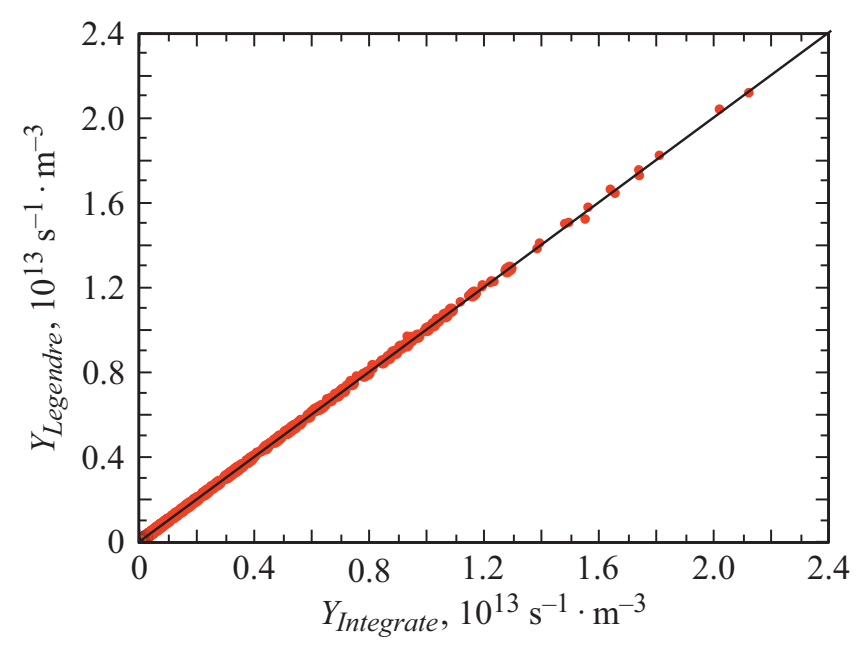

Рис. 2. Сравнение алгоритмов расчета нейтронного выхода (2) (ось абсцисс) и (3) (ось ординат) из разных точек плазменного шнура на токамаке Глобус-М2 для разрядов \#36620, 37067, 37069, 37070, 37893, 38089, 38095, 38516.

другом составляет $\sim 10-20 \%$ от суммарного. Дальнейшее увеличение тороидального магнитного поля и тока плазмы до проектных значений (1 Т и $500 \mathrm{kA})$, а также ввод в эксплуатацию второго инжектора нейтральных частиц (мощностью до $1 \mathrm{MW}$ ) приведет к увеличению этой доли до $\sim 30-50 \%$. В связи с этим при расчете полного нейтронного выхода возникает необходимость учитывать взаимодействие высокоэнергетических частиц друг с другом. Для этого возможно использование одного из двух приведенных алгоритмов: непосредственное интегрирование выражения (2) для удельной скорости реакции ядерного синтеза позволяет использовать данные с высоким уровнем статистических шумов и работать с функциями распределения любого вида, однако требует много времени на вычисления; разложение функций распределения по полиномам Лежандра (4) и суммирование тройных интегралов (3) - более быстрый алгоритм, однако он требует тщательного подбора используемой в разложении степени полинома Лежандра и параметра сглаживания функции распределения в случае, когда она получена методом МонтеКарло. При полуаналитическом подходе к вычислению функции распределения ионов, как, например, в работе [12], такой проблемы не возникает. Сложность алгоритма (2) может быть оценена как $O\left(N^{6}\right)$, где $N-$ размерность сетки функции распределения для одной пространственной точки по каждому из параметров, а алгоритма (3) - как $O\left(n N^{4}\right)$, где $n$ - выбранная степень полиномов Лежандра. Для характерных значений сетки, используемых для токамака Глобус-М2, скорость выполнения алгоритма (3) на два порядка выше, чем алгоритма (2). Как уже указывалось выше, сравнение этих алгоритмов показало хорошее согласие результатов, стандартное отклонение $\sigma<2 \%$.

\section{Финансирование работы}

Работа выполнена при финансовой поддержке Российского фонда фундаментальных исследований (грант 
18-32-20031 мол_а_вед). Использованы экспериментальные данные, полученные на Уникальной научной установке „Сферический токамак Глобус-М“, входящей в состав Федерального центра коллективного пользования „Материаловедение и диагностика в передовых технологиях“ (уникальный идентификатор RFMEFI62119X0021). Измерение базовых параметров плазменного разряда осуществлялось в рамках государственного задания Физико-технического института им. А.Ф. Иоффе Российской академии наук (№ 00402019-0023).

\section{Конфликт интересов}

Авторы заявляют, что у них нет конфликта интересов.

\section{Список литературы}

[1] V.B. Minaev, V.K. Gusev, N.V. Sakharov, Yu.V. Petrov, V.I. Varfolomeev, N.N. Bakharev, V.A. Belyakov, P.N. Brunkov, V.V. Bulanin, F.V. Chernyshev, V.I. Davydenko, V.V. Dyachenko, A.A. Kavin, S.A. Khitrov, N.A. Khromov, E.O. Kiselev, A.N. Konovalov, V.A. Kornev, G.S. Kurskiev, A.D. Melnik, A.B. Mineev, M.I. Mironov, I.V. Miroshnikov, A.N. Novokhatskii, K.Yu. Oshuev, M.I. Patrov, A.V. Petrov, V.A. Rozhansky, A.N. Saveliev, I.Yu. Senichenkov, P.B. Shchegolev, I.V. Shikhovtsev, A.D. Sladkomedova, V.V. Solokha, D.S. Sorokina, A.Yu. Telnova, V.A. Tokarev, S.Yu. Tolstyakov, A.Yu. Yashin, E.G. Zhilin, J. Phys.: Conf. Ser., 1094, 012001 (2018).

DOI: $10.1088 / 1742-6596 / 1094 / 1 / 012001$

[2] V.B. Minaev, V.K. Gusev, N.V. Sakharov, V.I. Varfolomeev, N.N. Bakharev, V.A. Belyakov, E.N. Bondarchuk, P.N. Brunkov, F.V. Chernyshev, V.I. Davydenko, V.V. Dyachenko, A.A. Kavin, S.A. Khitrov, N.A. Khromov, E.O. Kiselev, A.N. Konovalov, V.A. Kornev, G.S. Kurskiev, A.N. Labusov, A.D. Melnik, A.B. Mineev, M.I. Mironov, I.V. Miroshnikov, M.I. Patrov, Yu.V. Petrov, V.A. Rozhansky, A.N. Saveliev, I.Yu. Senichenkov, P.B. Shchegolev, O.N. Shcherbinin, I.V. Shikhovtsev, A.D. Sladkomedova, V.V. Solokha, V.N. Tanchuk, A.Yu. Telnova, V.A. Tokarev, S.Yu. Tolstyakov, E.G. Zhilin, Nucl. Fusion, 57, 066047 (2017). DOI: 10.1088/1741-4326/aa69e0

[3] V.K. Gusev, N.N. Bakharev, V.A. Belyakov, B.Ya. Ber, E.N. Bondarchuk, V.V. Bulanin, A.S. Bykov, F.V. Chernyshev, E.V. Demina, V.V. Dyachenko, P.R. Goncharov, A.E. Gorodetsky, E.Z. Gusakov, A.D. Iblyaminova, A.A. Ivanov, M.A. Irzak, E.G. Kaveeva, S.A. Khitrov, M.V. Khokhlov, N.A. Khromov, V.V. Kolmogorov, V.A. Kornev, S.V. Krasnov, G.S. Kurskiev, A.N. Labusov, S.A. Lepikhov, N.V. Litunovsky, I.V. Mazul, A.D. Melnik, V.V. Mikov, V.B. Minaev, A.B. Mineev, M.I. Mironov, I.V. Miroshnikov, E.E. Mukhin, A.N. Novokhatsky, A.D. Ovsyannikov, M.I. Patrov, A.V. Petrov, Yu.V. Petrov, V.A. Rozhansky, N.V. Sakharov, A.N. Saveliev, I.Yu. Senichenkov, V.Yu. Sergeev, P.B. Shchegolev, O.N. Shcherbinin, I.V. Shikhovtsev, V.S. Tanaev, V.N. Tanchuk, S.Yu. Tolstyakov, V.I. Varfolomeev, E.O. Vekshina, A.V. Voronin, S.P. Voskoboinikov, F. Wagner, A.Yu. Yashin, G.V. Zadvitskiy, A.P. Zakharov, R.Kh. Zalavutdinov, E.G. Zhilin, Nucl. Fusion, 55, 104016 (2015). DOI: $10.1088 / 0029-5515 / 55 / 10 / 104016$
[4] Н.Н. Бахарев, И.М. Балаченков, В.И. Варфоломеев, А.В. Воронин, В.К. Гусев, В.В. Дьяченко, М.В. Ильясова, Е.О. Киселев, А.Н. Коновалов, Г.С. Курскиев, А.Д. Мельник, В.Б. Минаев, И.В. Мирошников, А.Н. Новохацкий, М.И. Патров, Ю.В. Петров, Н.В. Сахаров, О.М. Скрекель, А.Ю. Тельнова, В.А. Токарев, С.Ю. Толстяков, Е.А. Тюхменева, Е.М. Хилькевич, Н.А. Хромов, Ф.В. Чернышев, И.Н. Чугунов, А.Е. Шевелев, П.Б. Щеголев, Физика плазмы, 46 (7), 579 (2020). DOI: 10.31857/S036729212007001X

[5] M.M. Larionova, I.V. Miroshnikov, V.K. Gusev, V.B. Minaev, M.I. Patrov, Yu.V. Petrov, N.V. Sakharov, P.B. Schegolev, A.Yu. Telnova, N.N. Bakharev, J. Phys.: Conf. Ser., 1400, 077018 (2019). DOI: 10.1088/1742-6596/1400/7/077018

[6] Г.С. Курскиев, С.Ю. Толстяков, А.А. Березуцкий, В.К. Гусев, М.М. Кочергин, В.Б. Минаев, Е.Е. Мухин, М.И. Патров, Ю.В. Петров, Н.В. Сахаров, В.В. Семёнов, П.В. Чернаков, ВАНТ. Сер. Термоядерный синтез, 2, 81 (2012). $\mathrm{http} / / /$ vant.iterru.ru/engvant_2012_2/6.pdf

[7] E.A. Tukhmeneva, S.Yu. Tolstyakov, G.S. Kurskiev, V.K. Gusev, V.B. Minaev, Yu.V. Petrov, N.V. Sakharov, A.Yu. Telnova, N.N. Bakharev, P.B. Shchegolev, E.O. Kiselev, Plasma Sci. Technol., 21, 105104 (2019). DOI: $10.1088 / 2058-6272 / a b 305 f$

[8] A. Pankin, D. McCune, R. Andre, G. Bateman, A. Kritz, Comput. Phys. Commun., 159, 157 (2004).

DOI: $10.1016 /$ j.cpc.2003.11.002

[9] E. Hirvijok., O. Asunta, T. Koskel, T. Kurki-Suonio, J. Miettunen, S. Sipilä, A. Snicker, S. Äkäslompolo, Comput. Phys. Commun., 185, 1310 (2014). DOI: 10.1016/j.cpc.2014.01.014

[10] N.N. Bakharev, F.V. Chernyshev, P.R. Goncharov, V.K. Gusev, A.D. Iblyaminova, V.A. Kornev, G.S. Kurskiev, A.D. Melnik, V.B. Minaev, M.I. Mironov, Nucl. Fusion., 55, 043023 (2015). DOI: $10.1088 / 0029-5515 / 55 / 4 / 043023$

[11] П.Р. Гончаров, ВАНТ. Сер. Термоядерный синтез, 38 (3), 22 (2015). http://vant.iterru.ru/vant_2015_3/3.pdf

[12] P.R. Goncharov, B.V. Kuteev, T. Ozaki, S. Sudo, Phys. Plasmas, 17, 112313 (2010). DOI: 10.1063/1.3505482 